\title{
Concordance between PIK3CA mutations in endoscopic biopsy and surgically resected specimens of esophageal squamous cell carcinoma
}

Ken Hatogai ${ }^{1,2,3}$, Satoshi Fujii ${ }^{2}$, Takashi Kojima ${ }^{1}$, Hiroyuki Daiko ${ }^{4}$, Toshihiko Doi ${ }^{1}$, Atsushi Ohtsu ${ }^{1,5}$, Atsushi Ochiai ${ }^{2}$, Yuichi Takiguchi ${ }^{3}$ and Takayuki Yoshino ${ }^{1 *}$

\begin{abstract}
Background: PIK3CA mutations are expected to be potential therapeutic targets for esophageal squamous cell carcinoma (ESCC). We aimed to clarify the concordance between PIK3CA mutations detected in endoscopic biopsy specimens and corresponding surgically resected specimens.

Methods: We examined five hotspot mutations in the PIK3CA gene (E542K, E545K, E546K, H1047R, and H1047L) in formalin-fixed and paraffin-embedded tissue sections of paired endoscopic biopsy and surgically resected specimens from 181 patients undergoing curative resection for ESCC between 2000 and 2011 using a Luminex technology-based multiplex gene mutation detection kit.

Results: Mutation analyses were successfully performed for both endoscopic biopsy and surgically resected specimens in all the cases. A PIK3CA mutation was detected in either type of specimen in 13 cases $(7.2 \%$, 95\% confidence interval: $3.9-12.0)$. The overall concordance rate, positive predictive value, and negative predictive value were $98.3 \%$ (178/181), 90.9\% (10/11), and 98.8\% (168/170), respectively. Among patients with a PIK3CA mutation detected in both types of specimens, the concordance between PIK3CA mutation genotypes was $100 \%$. There were three cases with a discordant mutation status between the types of specimens (PIK3CA mutation in surgically resected specimen and wild-type in biopsy specimen in two cases, and the opposite pattern in one case), suggesting possible intratumoral heterogeneity in the PIK3CA mutation status.

Conclusions: The PIK3CA mutation status was highly concordant between endoscopic biopsy and surgically resected specimens from the same patient, suggesting that endoscopic biopsy specimens can be clinically used to detect PIK3CA mutations in patients with ESCC.
\end{abstract}

Keywords: Esophageal squamous cell carcinoma, PIK3CA gene, Endoscopic biopsy, Concordance

\section{Background}

Squamous cell carcinoma is the predominant histological subtype of esophageal cancer in Asia, whereas adenocarcinomas predominate in the United Kingdom, some other Western European countries, and the United States [1]. To date, multidisciplinary treatment approaches for

\footnotetext{
* Correspondence: sfujii@east.ncc.go.jp; tyoshino@east.ncc.go.jp

${ }^{2}$ Division of Pathology, Exploratory Oncology Research \& Clinical Trial Center,

National Cancer Center, Kashiwa, Chiba, Japan

'Department of Gastroenterology and Gastrointestinal Oncology, National Cancer

Center Hospital East, 6-5-1, Kashiwanoha, Kashiwa, Chiba 277-8577, Japan

Full list of author information is available at the end of the article
}

esophageal cancer with different histological subtypes including surgery, chemotherapy, and radiation have been employed; however, the prognosis of these patients remains poor [2, 3]. For patients with metastatic or recurrent esophageal squamous cell carcinoma (ESCC), the available agents are quite limited. A combination of platinum agents and fluorouracil derivatives is commonly used as first-line chemotherapy, and taxanes are options for second-line chemotherapy [1, 4]. In addition, no molecular-target therapies have been established for the treatment of ESCC. Therefore, there is an unmet medical 
need for ESCC treatment, particularly for patients who are in good physical condition but who are refractory or intolerant to standard therapies.

The phosphoinositide 3-kinase (PI3K)-Akt-mammalian target of rapamycin (mTOR) pathway plays a pivotal role in cancer cell proliferation, and mutations in the PIK3CA gene are commonly found in various cancers regardless of histological subtypes [5]. More than $80 \%$ of PIK3CA mutations occur in two major regions: the helical domain (exon 9), and the kinase domain (exon 20); moreover, three mutations (E542K, E545K, and H1047R) have been regarded as hotspot mutations [6]. In a phase 1 trial evaluating an mTOR inhibitor, a case with advanced ESCC exhibited a partial response, although the PIK3CA mutation status was unknown [7]. In addition, PIK3CA mutations have been suggested to be a potential predictive biomarker for PI3K-Akt-mTOR inhibitors in a review of early phase clinical trials for the testing of such agents in various solid cancers [8]. In this report, a case with squamous cell head and neck carcinoma, which is genetically similar to ESCC, harboring a PIK3CA mutation (H1047R) demonstrated a partial response to a PI3K-Akt-mTOR inhibitor. The frequency of PIK3CA mutations in ESCC has been reported to range from 2.2 to $21 \%$ [9-16], whereas mutations in genes in the RAS-RAF pathway are very rare $[15,17,18]$. Accordingly, PIK3CA mutations may be a potential target molecule in ESCC treatment.

Previous studies investigating the frequency of PIK3CA mutations in ESCC used available clinical samples obtained from either surgically resected specimens or biopsy specimens [9-16]. Clarifying whether PIK3CA mutations from biopsy specimens can be detected in corresponding surgically resected specimens is important for the future clinical development of ESCC treatment. Therefore, the present study examined the frequency of PIK3CA mutations and the concordance between PIK3CA mutations detected in endoscopic biopsy specimens and those detected in corresponding surgically resected specimens in patients with ESCC.

\section{Methods}

\section{Patients}

Among 352 previously untreated patients with ESCC who underwent a curative-intent transthoracic esophagectomy with extended lymphadenectomy at the National Cancer Center Hospital East, Kashiwa, Japan, between January 2000 and December 2011, a total of 181 patients were enrolled according to the following selection criteria: (i) pathological $\mathrm{T}$ factor of at least $\mathrm{T} 1 \mathrm{~b}$, (ii) availability of paired samples of endoscopic biopsy and surgically resected specimens, (iii) patient age of $\leq 75$ years, (iv) absence of past or concurrent history of cancer, (v) adequate organ function, and (vi) absence of in-hospital death following surgery.

\section{Tissue samples}

Archival formalin-fixed and paraffin-embedded (FFPE) tissue sections of paired endoscopic biopsy and surgically resected specimens from the enrolled patients were used for DNA extraction. Thin tissue sections $(4 \mu \mathrm{m})$ cut from an FFPE tissue block were placed on microscopic slides and stained with hematoxylin and eosin (H\&E) for histological examination. Five unstained tissue sections $(10 \mu \mathrm{m})$ were also continuously cut from the same tissue block and were placed on a glass slide. The tumor histology was confirmed by a pathologist specializing in gastrointestinal cancer (SF) based on a microscopic examination of the H\&E-stained slides. For the endoscopic biopsy specimens, the entire biopsy specimen was manually microdissected after confirming that the ratio of tumor cells to whole cells was $>20 \%$ on slides stained with H\&E. For the surgically resected specimens, a tumor area with a small amount of stromal cells where the ratio of tumor cells to whole cells was $>50 \%$ on slides stained with H\&E was manually microdissected (Fig. 1). DNA extraction was performed using the heat-induced retrieval method, as described previously $[19,20]$.

\section{PIK3CA mutation analysis}

PIK3CA mutations were detected using a Luminex (xMAP) technology-based multiplex gene mutation detection kit (GENOSEARCH Mu-PACK; MBL, Nagoya, Japan), which was developed prior to the present study; an optimal concordance between the kit and the conventional direct sequencing method was confirmed previously [21]. A total of five PIK3CA mutations, including codon 542 (E542K), codon 545 (E545K), and codon 546 (E546K) in exon 9 and codon 1047 (H1047R, H1047L) in exon 20, were investigated. The lowest detection limit of the percentage of mutant allele was $5 \%$. The protocol details have been described previously [21]. Nextgeneration sequencing (NGS, Ion Ampliseq ${ }^{\mathrm{Tm}}$ Cancer Hotspot Panel v2; Life Technologies, Carlsbad, CA, USA) was additionally performed using DNA obtained from the same FFPE block for cases in which the mutation status of the endoscopic biopsy specimen and the surgically resected specimen differed when assessed using the method described above. In cases where NGS was not successfully performed, DNAs were extracted from two separate tumor portions of the same FFPE block as that used in the primary analysis, and PIK3CA mutations in each portion were measured using the kit.

\section{Statistical analysis}

The PIK3CA mutation frequency was determined as the proportion of specimens with a PIK3CA mutation among either all the endoscopic biopsy or all the surgically resected specimens. In addition to the mutation frequency, the overall concordance rate, positive concordance rate, negative 

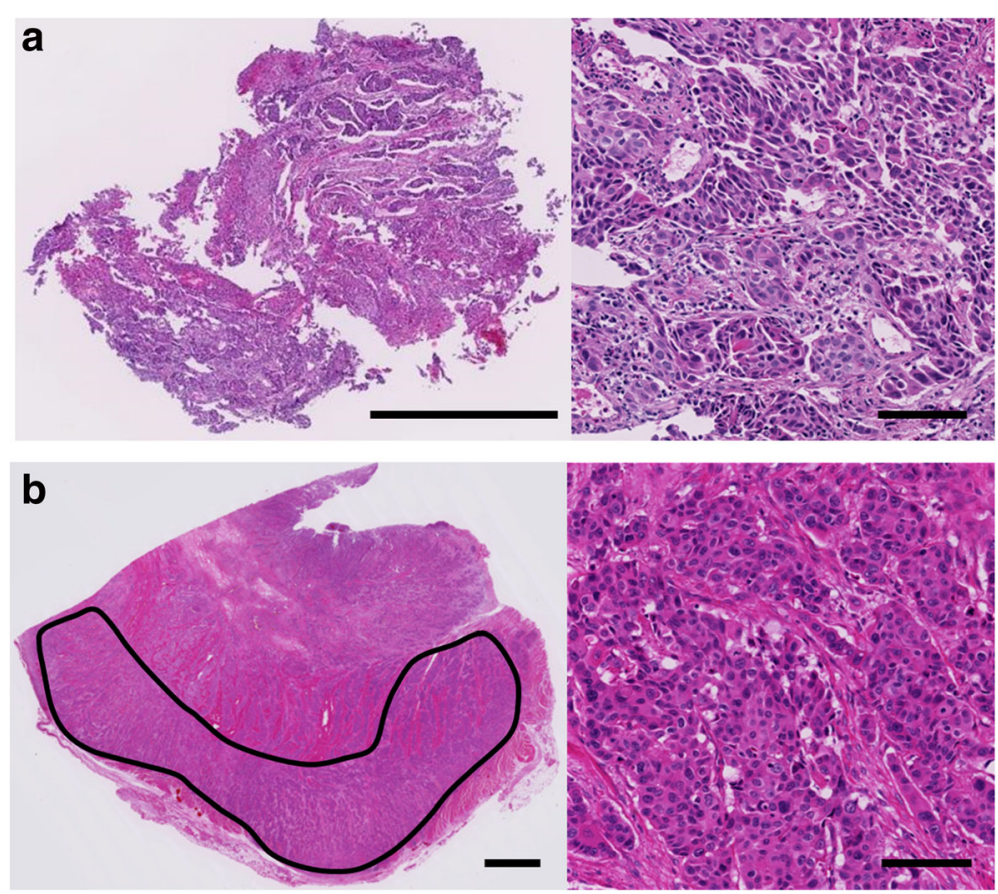

Fig. 1 Photomicrographs of a case harboring a PIK3CA mutation in both types of specimens (H1047R; Case No. 66). Paired endoscopic biopsy and surgically resected specimens show the same mutation. a The entire biopsy was manually microdissected (scale bar: $1 \mathrm{~mm}$ ). b The tumor area in the surgically resected specimen was marked (indicated by the solid line) and manually microdissected (scale bar: 2 mm). High-power views of each specimen are shown in the right panels (scale bar: $100 \mu \mathrm{m}$ )

concordance rate, positive predictive value, and negative predictive value of the endoscopic biopsy specimens, compared with the surgically resected specimens as a reference, were evaluated and presented with the $95 \%$ confidence intervals (CI) [22]. The associations between the PIK3CA mutation status and clinicopathological factors were assessed using the $t$-test for continuous variables and the chi-square test for categorical variables or the Fisher's exact test for dichotomous variables. All $P$ values were reported as two-sided, with a significance level of 0.05. All statistical analyses were performed using IBM SPSS statistics 20 (IBM Japan Ltd., Tokyo, Japan).

\section{Results}

\section{Patients}

The clinicopathological characteristics of the enrolled patients are shown in Table 1 . The mean age of the patients was 62.7 years, with the majority of the patients being male $(82.9 \%)$. Most of the patients had T2 or T3 disease, but patients with T1b disease $(28.2 \%)$ and curatively resected $\mathrm{T} 4$ disease $(2.2 \%)$ were included. One hundred twenty-five patients (68.5\%) had lymph node metastases, while $56(31.5 \%)$ did not. Eleven patients (6.1\%) had non-regional lymph node metastases (M1) but not distant organ metastases and were judged as Stage IV. In total, 362 samples from 181 patients were included in the concordance analysis.

\section{Frequency of PIK3CA mutations}

The median amount of extracted DNA was $11,268 \mathrm{ng}$ (interquartile range [IQR]: $6558-18,268$ ) for the surgically resected specimens and $2452 \mathrm{ng}$ (IQR: 1748-3352) for the biopsy specimens. For quality control, we checked the absorbance at $260 \mathrm{~nm}\left(\mathrm{~A}_{260}\right)$ and $280 \mathrm{~nm}$ $\left(\mathrm{A}_{280}\right)$, and the median $\mathrm{A}_{260} / \mathrm{A}_{280}$ scores were 1.91 (IQR: 1.85-1.98) and 1.93 (IQR: 1.88-1.97) for the endoscopic biopsy and surgically resected specimens, respectively. Mutation analyses were successfully performed for both the endoscopic biopsy and surgically resected specimens in all the cases. Among the endoscopic biopsy specimens, there were 11 cases with PIK3CA mutations: two with E542K, three with E545K, five with H1047R, and one with H1047L. Among the surgically resected specimens, there were 12 cases with PIK3CA mutations: one with E542K, four with E545K, six with H1047R, and one with H1047L. Overall, the PIK3CA mutation frequency based on a positive mutation status for either specimen was 7.2\% (13/181, 95\% CI: 3.9-12.0) (Table 2). Figure 1 shows micrographs of the ESCC case that harbored a PIK3CA mutation (H1047R) in both the biopsy and the surgically resected specimens. DNA was isolated from the entire biopsy specimen and the area marked by the solid line in the surgically resected specimen. No histological findings specific to cases with a PIK3CA mutation were observed in the present study. There were 
Table 1 Clinicopathological characteristics of the patients

\begin{tabular}{|c|c|c|}
\hline Characteristics & Number & Percent \\
\hline Age (mean $\pm S D)$ & $62.7 \pm 7.0$ & \\
\hline \multicolumn{3}{|l|}{ Gender } \\
\hline Male & 150 & 82.9 \\
\hline Female & 31 & 17.1 \\
\hline \multicolumn{3}{|l|}{ Smoking habit } \\
\hline Non-smoker & 33 & 18.2 \\
\hline Smoker & 148 & 81.8 \\
\hline \multicolumn{3}{|c|}{ Alcohol consumption } \\
\hline Non-drinker & 33 & 18.2 \\
\hline Drinker & 148 & 81.8 \\
\hline \multicolumn{3}{|l|}{ Location } \\
\hline Upper & 26 & 14.4 \\
\hline Middle & 83 & 45.9 \\
\hline Lower & 72 & 39.8 \\
\hline \multicolumn{3}{|l|}{ T factor } \\
\hline $1 b$ & 51 & 28.2 \\
\hline 2 & 22 & 12.2 \\
\hline 3 & 104 & 57.5 \\
\hline 4 & 4 & 2.2 \\
\hline \multicolumn{3}{|l|}{$\mathrm{N}$ factor } \\
\hline 0 & 57 & 31.5 \\
\hline 1 & 60 & 33.1 \\
\hline 2 & 48 & 26.5 \\
\hline 3 & 16 & 8.8 \\
\hline \multicolumn{3}{|l|}{ M factor } \\
\hline 0 & 170 & 93.9 \\
\hline 1 & 11 & 6.1 \\
\hline \multicolumn{3}{|l|}{ TNM stage } \\
\hline । & 30 & 16.6 \\
\hline$\|$ & 51 & 28.2 \\
\hline III & 89 & 49.2 \\
\hline IV & 11 & 6.1 \\
\hline \multicolumn{3}{|l|}{ Histological grade } \\
\hline W/D & 38 & 21.0 \\
\hline$M / D$ & 120 & 66.3 \\
\hline$P / D$ & 23 & 12.7 \\
\hline \multicolumn{3}{|c|}{ Lymphatic invasion } \\
\hline Absent & 89 & 49.2 \\
\hline Present & 92 & 50.8 \\
\hline \multicolumn{3}{|l|}{ Venous invasion } \\
\hline Absent & 36 & 19.9 \\
\hline Present & 145 & 80.1 \\
\hline
\end{tabular}

Abbreviations: $W / D$ well differentiated, $M / D$ moderately differentiated, $P / D$ poorly differentiated, $S D$ standard deviation
Table 2 Cases with PIK3CA mutations in either endoscopic biopsy or surgically resected specimens

\begin{tabular}{|c|c|c|c|c|c|}
\hline \multirow[b]{2}{*}{ Case } & \multicolumn{2}{|c|}{ Endoscopic biopsy } & \multicolumn{2}{|c|}{ Surgical resection } & \multirow{2}{*}{ T factor } \\
\hline & Mutation & $\overline{\text { Histology }}$ & Mutation & $\overline{\text { Histology }}$ & \\
\hline 8 & $\mathrm{E} 542 \mathrm{~K}$ & $M / D$ & Wild type & $M / D$ & T3 \\
\hline 50 & E545K & $M / D$ & E545K & $M / D$ & $\mathrm{~T} 1 \mathrm{~b}$ \\
\hline 61 & Wild type & $P / D$ & E545K & $M / D$ & $\mathrm{~T} 4$ \\
\hline 66 & H1047R & $M / D$ & H1047R & $P / D$ & T3 \\
\hline 75 & H1047R & $M / D$ & H1047R & $M / D$ & T3 \\
\hline 87 & E542K & $M / D$ & $\mathrm{E} 542 \mathrm{~K}$ & $M / D$ & T3 \\
\hline 111 & E545K & $M / D$ & E545K & $M / D$ & $\mathrm{~T} 3$ \\
\hline 114 & E545K & $M / D$ & E545K & $M / D$ & T3 \\
\hline 127 & H1047R & $M / D$ & H1047R & $M / D$ & T3 \\
\hline 132 & Wild type & $M / D$ & H1047R & $M / D$ & $\mathrm{~T} 1 \mathrm{~b}$ \\
\hline 140 & H1047L & $M / D$ & H1047L & $M / D$ & T3 \\
\hline 163 & H1047R & $M / D$ & H1047R & $M / D$ & T3 \\
\hline 166 & H1047R & $M / D$ & H1047R & W/D & $\mathrm{T} 1 \mathrm{~b}$ \\
\hline
\end{tabular}

Abbreviations: $W / D$ well differentiated, $M / D$ moderately differentiated, $P / D$ poorly differentiated

three cases with discordant results between the endoscopic biopsy and the surgically resected specimens.

The correlations between clinicopathological factors and the PIK3CA mutation status as detected using DNA extracted from either the endoscopic biopsy or surgically resected specimens are presented in Table 3. No clear differences were observed between the PIK3CA mutation status and the clinicopathological factors that were examined.

\section{Concordance analysis}

As shown in Table 4, 168 cases and 10 cases were determined to have a wild-type and a mutant-type, respectively, in both the endoscopic biopsy and surgically resected specimens. In contrast, a concordant result was not achieved in the remaining three cases. The overall concordance rate for the PIK3CA mutation status between the endoscopic biopsy and surgically resected specimens was $98.3 \%([168+10] / 181$, 95\% CI: $95.2-$ 99.7). The positive and negative concordance rates were 83.3\% (10/12, 95\% CI: 51.6-97.9) and 99.4\% (168/169, 95\% CI: 96.7-99.9), respectively. The positive and negative predictive values were $90.9 \%(10 / 11,95 \% \mathrm{CI}$ : $58.7-99.8)$ and $98.8 \%$ (168/170, 95\% CI: 95.8-99.9), respectively.

The PIK3CA mutation genotypes in the endoscopic biopsy and surgically resected specimens obtained for the ten cases that exhibited a PIK3CA mutation in both specimen types are compared in Table 5. The concordance of the PIK3CA mutation genotype was $100 \%$ (10/10, 95\% CI: $74 \%-100 \%)$. 
Table 3 Relationship between clinicopathological characteristics and PIK3CA mutation status

\begin{tabular}{|c|c|c|c|c|c|}
\hline \multirow[t]{3}{*}{ Characteristics } & \multicolumn{4}{|c|}{ PIK3CA mutation status } & \multirow{3}{*}{$P$ value } \\
\hline & \multicolumn{2}{|l|}{ Wild-type } & \multicolumn{2}{|l|}{ Mutant } & \\
\hline & Number & $\%$ & Number & $\%$ & \\
\hline Mean age $\pm S D$ & $62.8 \pm 7.1$ & & $61.9 \pm 5.6$ & & 0.643 \\
\hline Gender & & & & & 0.469 \\
\hline Male & 140 & 83.3 & 10 & 76.9 & \\
\hline Female & 28 & 16.7 & 3 & 23.1 & \\
\hline Smoking status & & & & & 0.468 \\
\hline Non-smoker & 32 & 19.0 & 1 & 7.7 & \\
\hline Smoker & 136 & 81.0 & 12 & 92.3 & \\
\hline Alcohol consumption & & & & & 1.000 \\
\hline Non-drinker & 14 & 8.3 & 1 & 7.7 & \\
\hline Drinker & 154 & 91.7 & 12 & 92.3 & \\
\hline Location & & & & & 1.000 \\
\hline Upper/Middle & 101 & 60.1 & 8 & 61.5 & \\
\hline Lower & 67 & 39.9 & 5 & 38.5 & \\
\hline T factor & & & & & 0.247 \\
\hline $1-2$ & 70 & 41.7 & 3 & 23.1 & \\
\hline $3-4$ & 98 & 58.3 & 10 & 76.9 & \\
\hline Lymph node metastases & & & & & 1.000 \\
\hline Absent & 52 & 31.0 & 4 & 30.8 & \\
\hline Present & 116 & 69.0 & 9 & 69.2 & \\
\hline TNM stage & & & & & 0.775 \\
\hline$|-| \mid$ & 76 & 45.2 & 5 & 38.5 & \\
\hline III-IV & 92 & 54.8 & 8 & 61.5 & \\
\hline Histological differentiation & & & & & 1.000 \\
\hline W/D, M/D & 146 & 86.9 & 12 & 92.3 & \\
\hline$P / D$ & 22 & 13.1 & 1 & 7.7 & \\
\hline Lymphatic invasion & & & & & 0.400 \\
\hline Absent & 81 & 48.2 & 8 & 61.5 & \\
\hline Present & 87 & 51.8 & 5 & 38.5 & \\
\hline Venous invasion & & & & & 0.470 \\
\hline Absent & 35 & 20.8 & 1 & 7.7 & \\
\hline Present & 133 & 79.2 & 12 & 92.3 & \\
\hline
\end{tabular}

Abbreviations: $S D$ standard deviation, $W / D$ well differentiated, $M / D$ moderately differentiated, $P / D$ poorly differentiated

Details of discrepancy of PIK3CA mutation status between endoscopic biopsy and surgically resected specimens

The details of the three cases with a discordant PIK3CA mutation status were as follows: two cases had mutationpositive surgical specimens but exhibited a wild-type profile for their biopsy specimens (Case No. 61 and No. 132), while the opposite pattern was observed in one case (Case No. 8; Table 2). We performed NGS for these three cases; however, the DNA amplification was incomplete and the sequencing was not successful in both Case No. 8 and
Table 4 Concordance of PIK3CA mutation status between endoscopic biopsy and surgically resected specimens

\begin{tabular}{|c|c|c|c|c|}
\hline & & \multicolumn{3}{|c|}{$\begin{array}{l}\text { Surgically resected } \\
\text { specimens } \\
\text { (reference method) }\end{array}$} \\
\hline & & Wild type & Mutant type & Total \\
\hline \multirow{3}{*}{$\begin{array}{l}\text { Endoscopic biopsy } \\
\text { specimens (trial method) }\end{array}$} & Wild type & 168 & 2 & 170 \\
\hline & Mutant type & 1 & 10 & 11 \\
\hline & Total & 169 & 12 & 181 \\
\hline \multicolumn{2}{|l|}{ Overall concordance rate } & \multicolumn{3}{|c|}{$98.3 \%$ (95\% Cl: 95.2-99.7) } \\
\hline \multicolumn{2}{|l|}{ Positive concordance rate } & \multicolumn{3}{|c|}{$83.3 \%$ (95\% Cl: 51.6-97.9) } \\
\hline \multicolumn{2}{|l|}{ Negative concordance rate } & \multicolumn{3}{|c|}{ 99.4\% (95\% Cl: 96.7-99.9) } \\
\hline \multicolumn{2}{|l|}{ Positive predictive value } & \multicolumn{3}{|c|}{$90.9 \%$ (95\% Cl: 58.7-99.8) } \\
\hline \multicolumn{2}{|l|}{ Negative predictive value } & \multicolumn{3}{|c|}{ 98.8\% (95\% Cl: 95.8-99.9) } \\
\hline
\end{tabular}

Abbreviations: $\mathrm{Cl}$ confidence interval

Case No. 61. In Case No. 132, NGS revealed a PIK3CA mutation in H1047R with a mutant allele frequency of $4.5 \%$ in the surgically resected specimen; no PIK3CA mutations were detected in the endoscopic biopsy specimen. In Case No. 8 and Case No. 61, PIK3CA mutations were detected in only one portion and were not detected in the other when two separate tumor portions from the same FFPE block were analyzed (Fig. 2).

\section{Discussion}

To the best of our knowledge, this is the first report to investigate the concordance of the PIK3CA mutation status between endoscopic biopsy and surgically resected specimens using FFPE tissues from patients with ESCC. In the present study, we demonstrated that, although not frequent, a certain proportion of patients with ESCC harbored PIK3CA mutations, and the mutation statuses of the two types of specimens were highly concordant.

Among the five hotspot mutations assessed in the present study, E542K, E545K and E546K are located in exon 9, corresponding to the helical domain, and H1047R and H1047L are located in exon 20, corresponding to the kinase domain. The mutations in both of these domains

Table 5 Concordance of PIK3CA mutation genotypes between endoscopic biopsy and surgically resected specimens in cases exhibiting a PIK3CA mutation in both specimen types

\begin{tabular}{lllllll}
\hline & & $\begin{array}{l}\text { Surgically resected } \\
\text { specimens } \\
\text { (reference method) }\end{array}$ & \\
\cline { 2 - 7 } & & E542K & E545K & E546Q & H1047R & H1047L \\
\hline $\begin{array}{l}\text { Endoscopic biopsy } \\
\text { specimens }\end{array}$ & E542K & 1 & 0 & 0 & 0 & 0 \\
& E545K & 0 & 3 & 0 & 0 & 0 \\
& E546Q & 0 & 0 & 0 & 0 & 0 \\
& H1047R & 0 & 0 & 0 & 5 & 0 \\
& H1047L & 0 & 0 & 0 & 0 & 1 \\
\hline
\end{tabular}




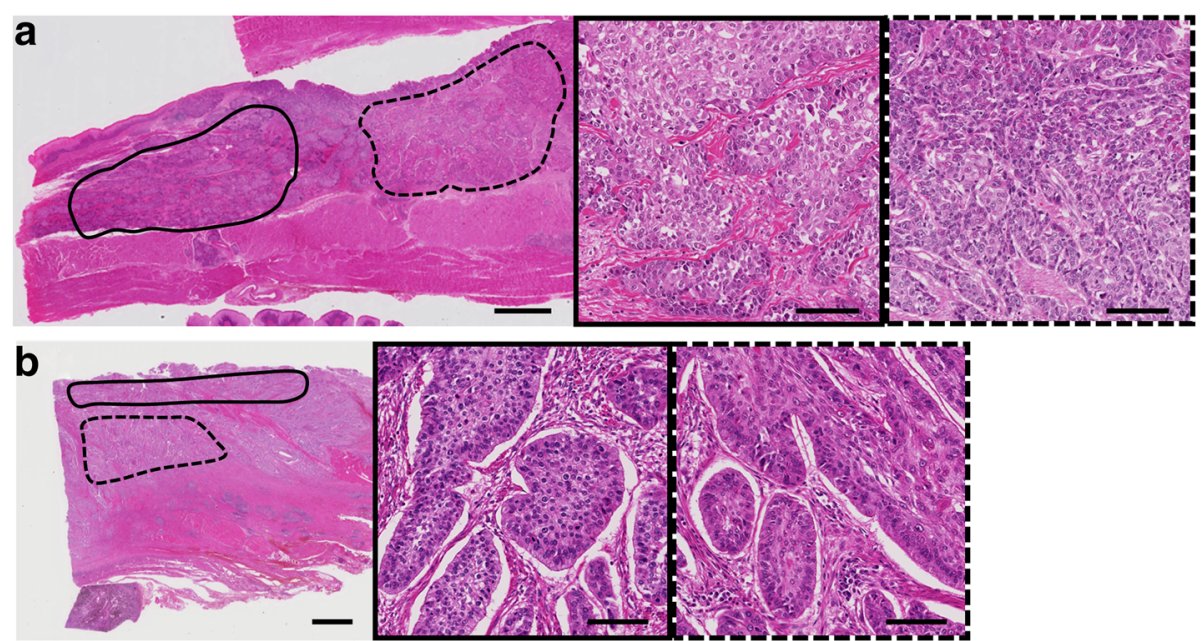

Fig. 2 Photomicrographs of two cases with a heterogeneous PIK3CA mutation status. A PIK3CA mutation was detected in the portions marked by the dotted lines, but not in the portion marked by the solid lines (scale bar: 2 mm). a Case No. 8 and $\mathbf{b}$ Case No. 61. High-power views of each portion indicated by the solid and dotted lines are shown in the insets (scale bar: $100 \mu \mathrm{m}$ )

increase the kinase activity of PI3K and activate the PI3KAkt-mTOR pathway, resulting in the activation of cell signaling and the promotion of cell growth and invasion $[23,24]$. The present study showed no significant association between the PIK3CA mutation status and the clinicopathological characteristics of the ESCC cases, suggesting that an examination of the clinicopathological factors prior to genetic analysis might not be capable of predicting the presence of a PIK3CA mutation. In other words, ESCC patients with a PIK3CA mutation constitute a subgroup only in terms of the applicability of a PI3K inhibitor, since emerging evidence suggests that patients with PIK3CA-mutated cancer might benefit from treatment with PI3K inhibitors $[8,25]$.

The high overall concordance rate $(98.3 \%)$ between endoscopic biopsy and surgically resected specimens observed in this study suggests that PIK3CA mutations are homogeneously distributed in the primary tumor in most cases. Although the intratumoral heterogeneity of the PIK3CA mutation status has not yet been investigated for ESCC, the results of our study are in line with the homogeneous distribution of the PIK3CA mutation status in the primary tumor observed in colorectal and breast cancers $[26,27]$. In colorectal cancer, the concordance rate of the KRAS mutation status between endoscopic biopsy and surgically resected specimens is high [28], similar to that of the PIK3CA mutation status in ESCC demonstrated in the present study, and endoscopic biopsy specimens are used for the molecular analysis of KRAS mutations to evaluate the clinical indications for anti-epidermal growth factor receptor antibody therapy. These findings suggest that FFPE clinical samples obtained from endoscopic biopsies are applicable to the identification of PIK3CA mutations in ESCC. In contrast, the discordant mutation status between endoscopic biopsy and surgically resected specimens that was observed in three cases may be attributable to intratumoral heterogeneity. Because endoscopic biopsy specimens represent a limited and superficial sampling of the primary tumor, intratumoral heterogeneity can be an obstacle to establishing a precise biomarker diagnosis. Obtaining multiple endoscopic biopsy samples from primary tumors may improve the likelihood of detecting a mutation and may minimize potential mutational discordances.

The PIK3CA mutation frequency of $7.2 \%$ observed in this study is based on Luminex (xMAP) technology targeting five hotspot mutations in the PIK3CA gene with a detection limit of $5 \%$ and is compatible with the COSMIC database published by the Sanger institute (9.5\%), and with those of the previous studies ranging from 2.2 to $21 \%$ [9-16]. The variation in mutation frequency among these studies is thought to be mainly attributable to differences in the methods used to detect PIK3CA mutations as well as differences in the patient cohorts, with factors such as disease stage, prior therapy, and ethnicity playing major roles. The frequency of PIK3CA mutations has been reported to be $2.2 \%-7.7 \%$ using direct sequencing, $11.5 \%-21 \%$ using pyrosequencing or other high-sensitivity methods, and 4.5\%-9.0\% using NGS $[9-12,14-16,29,30]$. The results of the present study were likely influenced by the sensitivity of the mutation testing. Collecting a sufficient number of cancer cells and excluding non-cancerous cells from biopsy specimens is difficult using manual microdissection for DNA extraction because the original size of the biopsy specimens is considerably smaller than that of the surgically resected specimens. One strategy is to use a detection method with a higher sensitivity, thereby reducing the risk of missing a relatively small fraction of cancer 
cells carrying a PIK3CA mutation. However, the relationship between the proportion of cancer cells with a PIK3CA mutation and biological differences has not yet been reported, and the relationship between the proportion of cancer cells with a PIK3CA mutation and differences in the response to PI3K inhibitors for any cancer type, including ESCC, remains unknown. Importantly, the present study revealed a case in which a PIK3CA mutation was observed in a biopsy specimen, but the wild-type was observed in the surgically resected specimen. This event suggests the existence of another problem in the detection of PIK3CA mutations: that is, heterogeneity. In addition to the sensitivity of mutation testing, tumor heterogeneity is also likely to influence the detection sensitivity. Since the efficacy of agents inhibiting the PI3K-Akt-mTOR pathway has been demonstrated clinically, further investigation of the optimal detection method and its detection limit is needed to ensure that patients who might benefit from such treatment are accurately identified.

Recently, phase 1, phase 2, and phase 3 clinical trials examining a number of PI3K inhibitors have begun for patients with various types of cancer [31, 32]. Among these agents, the clinical efficacy of buparlisib (BKM120), a pan-PI3K inhibitor, has been demonstrated in breast cancer patients in a phase 3 trial, and the presence of a PIK3CA mutation was shown to predict a response to this agent [25]. Several phase 2 trials examining buparlisib in ESCC patients are also currently ongoing (registration ID: NCT01806649, UMIN000011217). Additional biomarker studies performed during these clinical trials may reveal whether the PIK3CA mutation can be used as a biomarker to predict the efficacy of PI3K inhibitors in patients with ESCC.

Although heterogeneity in the PIK3CA mutation status between primary tumors and corresponding lymph nodes or distant organ metastases is reportedly rare for colorectal and breast cancers, limited information is available with regard to ESCC. For the development of therapies targeting the PIK3CA mutation in patients with ESCC, the potential for heterogeneity between primary tumors and metastases must be further investigated.

\section{Conclusion}

The detection of PIK3CA mutations could be used to define a subset of patients who may be potential candidates for treatment with inhibitors of the PI3K-AktmTOR pathway. The PIK3CA mutation status was highly concordant between endoscopic biopsy and surgically resected specimens in patients with ESCC, suggesting that endoscopic biopsy specimens are clinically applicable for the detection of PIK3CA mutations.

\section{Abbreviations}

Cl: Confidence intervals; ESCC: Esophageal squamous cell carcinoma;

FFPE: Formalin-fixed and paraffin-embedded; H\&E: Hematoxylin-eosin; IQR: Interquartile range; mTOR: Mammalian target of rapamycin;

PI3K: Phosphoinositide 3-kinase

\section{Acknowledgements}

We are grateful to Ms. Mari Takahashi for technical assistance and to Mr. Shogo Nomura for statistical advice.

\section{Funding}

This study was supported by research funding from National Cancer Center Hospital East.

\section{Availability of data and materials}

The dataset supporting the conclusions of this article is included within the article.

\section{Authors' contributions}

$\mathrm{KH}, \mathrm{SF}, \mathrm{TK}$, and TY contributed to the conception and design of the study. $\mathrm{KH}$ and SF performed the immunohistochemical study and gene mutation analysis. TK, HD, TD, OA (Ohtsu) and TY provided clinical data and helped collect the tumor tissues. KH, SF, TK, and TY performed the statistical analysis. $\mathrm{KH}, \mathrm{SF}, \mathrm{TK}$, and TY drafted the manuscript. YT and AO (Ochiai) coordinated the study and helped to draft the manuscript. All the authors have read and approved the final manuscript.

\section{Competing interests}

The authors declare that they have no competing interests.

\section{Consent for publication}

Not applicable.

\section{Ethics approval and consent to participate \\ The study protocol was approved by the institutional review board of the National Cancer Center in October 2014 (2014-135). The study was performed according to the Epidemiological Study Guideline of Ministry of Health, Labour and Welfare in Japan. Tissue samples were collected from patients who received esophagectomy for ESCC and provided written informed consent for use of the archival tissues. We described the study design on the National Cancer Center's website and gave the relatives of the deceased patients the opportunity to decline participation in the present study.}

\section{Author details}

'Department of Gastroenterology and Gastrointestinal Oncology, National Cancer Center Hospital East, 6-5-1, Kashiwanoha, Kashiwa, Chiba 277-8577, Japan. ${ }^{2}$ Division of Pathology, Exploratory Oncology Research \& Clinical Trial Center, National Cancer Center, Kashiwa, Chiba, Japan. ${ }^{3}$ Department of Medical Oncology, Graduate School of Medicine, Chiba University, Chuo-ku, Chiba, Japan.

${ }^{4}$ Department of Esophageal Surgery, National Cancer Center Hospital East, Kashiwa, Chiba, Japan. ${ }^{5}$ Exploratory Oncology Research \& Clinical Trial Center, National Cancer Center, Kashiwa, Japan.

Received: 12 December 2015 Accepted: 20 December 2016 Published online: 09 January 2017

\section{References}

1. Pennathur A, Gibson MK, Jobe BA, et al. Oesophageal carcinoma. Lancet. 2013;381:400-12.

2. Enzinger PC, Mayer RJ. Esophageal cancer. N Engl J Med. 2003;349:2241-52.

3. Sjoquist KM, Burmeister BH, Smithers BM, et al. Survival after neoadjuvant chemotherapy or chemoradiotherapy for resectable oesophageal carcinoma: an updated meta-analysis. Lancet Oncol. 2011;12:681-92.

4. Thallinger CM, Raderer M, Hejna M. Esophageal cancer: a critical evaluation of systemic second-line therapy. J Clin Oncol. 2011;29:4709-14.

5. Courtney KD, Corcoran RB, Engelman JA. The PI3K pathway as drug target in human cancer. J Clin Oncol. 2010;28:1075-83.

6. Samuels $Y$, Wang $Z$, Bardelli A, et al. High frequency of mutations of the PIK3CA gene in human cancers. Science. 2004;304:554.

7. Okamoto I, Doi T, Ohtsu A, et al. Phase I clinical and pharmacokinetic study of RAD001 (everolimus) administered daily to Japanese patients with advanced solid tumors. Jpn J Clin Oncol. 2010;40:17-23. 
8. Janku F, Hong DS, Fu S, et al. Assessing PIK3CA and PTEN in early-phase trials with PI3K/AKT/mTOR inhibitors. Cell Rep. 2014;6:377-87.

9. Akagi l, Miyashita M, Makino $\mathrm{H}$, et al. Overexpression of PIK3CA is associated with lymph node metastasis in esophageal squamous cell carcinoma. Int J Oncol. 2009;34:767-75.

10. Hou J, Jiang D, Zhang J, et al. Frequency, characterization, and prognostic analysis of PIK3CA gene mutations in Chinese esophageal squamous cell carcinoma. Hum Pathol. 2014;45:352-8.

11. Maeng $\mathrm{CH}$, Lee J, van Hummelen $\mathrm{P}$, et al. High-throughput genotyping in metastatic esophageal squamous cell carcinoma identifies phosphoinositide-3-kinase and BRAF mutations. PLoS One. 2012;7, e41655.

12. Mori $\mathrm{R}$, Ishiguro $\mathrm{H}$, Kimura $\mathrm{M}$, et al. PIK3CA mutation status in Japanese esophageal squamous cell carcinoma. J Surg Res. 2008;145:320-6.

13. Phillips WA, Russell SE, Ciavarella ML, et al. Mutation analysis of PIK3CA and PIK3CB in esophageal cancer and Barrett's esophagus. Int J Cancer. 2006; 118:2644-6.

14. Shigaki H, Baba Y, Watanabe M, et al. PIK3CA mutation is associated with a favorable prognosis among patients with curatively resected esophageal squamous cell carcinoma. Clin Cancer Res. 2013;19:2451-9.

15. Song $Y, L i L$, Ou Y, et al. Identification of genomic alterations in oesophageal squamous cell cancer. Nature. 2014;509:91-5.

16. Wang $L$, Shan L, Zhang S, et al. PIK3CA gene mutations and overexpression: implications for prognostic biomarker and therapeutic target in Chinese esophageal squamous cell carcinoma. PLoS One. 2014;9, e103021.

17. Gonzaga IM, Soares-Lima SC, de Santos PT, et al. Alterations in epidermal growth factor receptors 1 and 2 in esophageal squamous cell carcinomas. BMC Cancer. 2012;12:569.

18. Shigaki $\mathrm{H}$, Baba $Y$, Watanabe $M$, et al. KRAS and BRAF mutations in 203 esophageal squamous cell carcinomas: pyrosequencing technology and literature review. Ann Surg Oncol. 2013;20 Suppl 3:S485-91.

19. Fukushima Y, Yanaka S, Murakami K, et al. High-throughput screening method of KRAS mutations at codons 12 and 13 in formalin-fixed paraffinembedded tissue specimens of metastatic colorectal cancer. Gan To Kagaku Ryoho. 2011;38:1825-35.

20. Gilbert MT, Haselkorn T, Bunce M, et al. The isolation of nucleic acids from fixed, paraffin-embedded tissues-which methods are useful when? PLoS One. 2007;2, e537.

21. Bando H, Yoshino T, Shinozaki E, et al. Simultaneous identification of 36 mutations in KRAS codons 61 and 146, BRAF, NRAS, and PIK3CA in a single reaction by multiplex assay kit. BMC Cancer. 2013;13:405.

22. Gonen M. Analyzing reciever operating characteristic curves with SAS. Cary: SAS Institute; 2007. p. 5-14.

23. Karakas B, Bachman KE, Park BH. Mutation of the PIK3CA oncogene in human cancers. Br J Cancer. 2006:94:455-9.

24. Engelman JA. Targeting PI3K signalling in cancer: opportunities, challenges and limitations. Nat Rev Cancer. 2009:9:550-62.

25. Baselga J, Im S-A, Iwata H, et al. PIK3CA status in circulating tumor DNA (ctDNA) predicts efficacy of buparlisib (BUP) plus fulvestrant (FULV) in postmenopausal women with endocrine-resistant HR+/HER2- advanced breast cancer (BC): First results from the randomized, phase III BELLE-2 trial. San Antonio: The San Antonio Breast Cancer Symposium; 2015. Abstract S6-01.

26. Baldus SE, Schaefer KL, Engers R, et al. Prevalence and heterogeneity of KRAS, BRAF, and PIK3CA mutations in primary colorectal adenocarcinomas and their corresponding metastases. Clin Cancer Res. 2010;16:790-9.

27. Kalinsky K, Heguy A, Bhanot UK, et al. PIK3CA mutations rarely demonstrate genotypic intratumoral heterogeneity and are selected for in breast cancer progression. Breast Cancer Res Treat. 2011;129:635-43.

28. Krol LC, $t$ Hart NA, Methorst N, et al. Concordance in KRAS and BRAF mutations in endoscopic biopsy samples and resection specimens of colorectal adenocarcinoma. Eur J Cancer. 2012;48:1108-15.

29. Gao YB, Chen ZL, Li JG, et al. Genetic landscape of esophageal squamous cell carcinoma. Nat Genet. 2014;46:1097-102.

30. Lin DC, Hao JJ, Nagata Y, et al. Genomic and molecular characterization of esophageal squamous cell carcinoma. Nat Genet. 2014;46:467-73.

31. Porta C, Paglino C, Mosca A. Targeting PI3K/Akt/mTOR Signaling in Cancer. Front Oncol. 2014:4:64

32. Stark AK, Sriskantharajah S, Hessel EM, et al. PI3K inhibitors in inflammation, autoimmunity and cancer. Curr Opin Pharmacol. 2015;23:82-91.

\section{Submit your next manuscript to BioMed Central and we will help you at every step:}

- We accept pre-submission inquiries

- Our selector tool helps you to find the most relevant journal

- We provide round the clock customer support

- Convenient online submission

- Thorough peer review

- Inclusion in PubMed and all major indexing services

- Maximum visibility for your research

Submit your manuscript at www.biomedcentral.com/submit
Biomed Central 